\title{
Development of compound semiconductor detectors at ESA
}

\author{
D H Lumb, A Owens, M Bavdaz, T Peacock \\ Advanced Concepts and Science Payloads Office, European Space Agency, ESTEC, Keplerlaan 1, 2200AG Noordwijk, The \\ Netherlands
}

\begin{abstract}
Some examples of space-borne applications that require improvements in detector technology compared with conventional Si and Ge designs are described. Properties of compound semiconductors are noted, and a range of different detector developments are briefly reviewed. Material fabrication improvements for several compound semiconductors have resulted in near Fano-limited performance.
\end{abstract}

Keywords Compound semiconductors, X-ray detection, Gamma-ray detection

\section{Introduction}

The group IV elements Silicon and Germanium are by far the most widely used detector semiconductors, but some compound semiconductor materials are being developed for special applications. In P-I-N semiconductor detectors the depletion depth is inversely proportional to net electrical impurity concentration, and since photon counting efficiency is also dependent on the thickness of the material, large volumes of very pure material are needed to ensure high counting efficiency for high energy photons. Prior to the mid-1970's the required purity levels of Si and Ge could be achieved only by counter-doping p-type crystals with the n-type impurity, lithium, in a process known as lithium-ion drifting. Although this process is still widely used in the production of $\mathrm{Si}(\mathrm{Li})$ X-ray detectors, it is no longer required for germanium detectors since sufficiently pure crystals have been available for more than 25 years. As a practical limitation, both $\mathrm{Ge}$ and $\mathrm{Si}$ photon detectors must be cooled in order to reduce the thermal charge carrier generation (and associated noise) to an acceptable level. Nevertheless, and particularly for space-borne observatories, silicon CCDs (charge coupled devices) have revolutionized imaging spectroscopy at energies $<10 \mathrm{keV}$ despite their modest photon stopping power [1].
In astrophysics, the energy band from 10 to 100 's keV has resisted development, in part due to lack of focusing techniques, but also to these detector limitations. This energy band represents an important transition between thermal and non-thermal astrophysical processes, as well as being of vital importance in medical radiology. These important scientific drivers have motivated much of the research into procuring compound semiconductor detectors.

Room temperature properties of some semiconductor materials are summarised in Table 1. Compounds employing III-V or II-VI group elements have larger energy gaps $(\sim 1.3$ to $3 \mathrm{eV}$ ) and atomic numbers from $\sim 30$ to $\sim 90$. The latter feature allows for greater stopping power, and the larger bandgap reduces requirements for cooling, while still being significantly less than for gaseous detectors, so that good energy resolution remains possible. Further advantages for space-borne applications accrue: larger stopping power implies thinner detectors that enhances radiation resistance (which is a bulk effect that scales with volume) and lowers leakage current. This radiation tolerance is further enhanced because the energy of defect formation in wide gap semiconductors is increased compared with the elemental semiconductors.

These advantages have not been fully realized despite many years of intense research. The basic problem remains the difficulty in growing detector grade material that is both chemically pure and structurally 
perfect in crystallinity and stochiometry. The Advanced Concepts and Science Payloads Office of ESA has been working for a number of years to produce monolithic and array compound semiconductor (CS) detectors for future space missions. Considerable progress has been made towards obtaining near Fanolimited performance, following a detailed material science and technology development programme. In some cases performance already exceeds that of $\mathrm{Si}$ or Ge for certain applications. We briefly highlight important results for some of these detectors, especially where they illustrate some common issues affecting CS detectors for other applications.

\begin{tabular}{|l|l|l|l|l|l|l|l|}
\hline Material & $\begin{array}{l}\text { Atomic } \\
\text { Number }\end{array}$ & $\begin{array}{l}\text { Density } \\
\left(\mathrm{g} \mathrm{cm}^{-3}\right)\end{array}$ & $\begin{array}{l}\text { Band gap } \\
(\mathrm{eV})\end{array}$ & $\begin{array}{l}\mathrm{E}_{\text {pair }} \\
(\mathrm{eV})\end{array}$ & $\begin{array}{l}\text { Resistivity } \\
(\Omega-\mathrm{cm})\end{array}$ & $\begin{array}{l}\mu \tau(\mathrm{e}) \text { Product } \\
\left(\mathrm{cm}^{2} / \mathrm{V}\right)\end{array}$ & $\begin{array}{l}\mu \tau(\mathrm{h}) \text { Product } \\
\left(\mathrm{cm}^{2} / \mathrm{V}\right)\end{array}$ \\
\hline $\mathrm{Si}$ & 14 & 2.33 & 1.12 & 3.63 & $>10^{4}$ & $2.710^{-2}$ & $9.610^{-3}$ \\
\hline $\mathrm{Ge}$ & 32 & 5.32 & 0.66 & 2.9 & 50 & $>1$ & $>1$ \\
\hline $\mathrm{GaAs}$ & 31,33 & 5.32 & 1.43 & 4.2 & $10^{7}$ & $810^{-5}$ & $410^{-6}$ \\
\hline $\mathrm{InP}$ & 49,15 & 4.78 & 1.43 & 4.2 & $10^{6}$ & $510^{-6}$ & $<210^{-5}$ \\
\hline $\mathrm{CdTe}$ & 48,52 & 6.2 & 1.44 & 4.43 & $10^{9}$ & $3.310^{-3}$ & $2.610^{-4}$ \\
\hline $\mathrm{Cd}_{0.9} \mathrm{Zn}_{0.1} \mathrm{Te}$ & $48,30,52$ & 5.8 & 1.57 & 4.64 & $10^{11}$ & $710^{-3}$ & $910^{-5}$ \\
\hline $\mathrm{PbI}_{2}$ & 82,53 & 6.2 & $2.3-2.5$ & 4.9 & $10^{12}$ & $810^{-6}$ & $910^{-7}$ \\
\hline $\mathrm{HgI}_{2}$ & 80,53 & 6.4 & 2.13 & 4.2 & $10^{13}$ & $10^{-4}$ & $410^{-5}$ \\
\hline $\mathrm{TlBr}^{13}$ & 81,35 & 7.56 & 2.68 & 6.5 & $10^{12}$ & $310^{-5}$ & $1.510^{-6}$ \\
\hline
\end{tabular}

Table 1 Typical room temperature properties of some wide band gap semiconductors that are under development for hard X-ray and $\gamma$-ray detectors

\section{Applications}

\subsection{Planetary Surface Imaging}

The next approved mission in the ESA planetary science programme is the BepiColombo [2] project to Mercury expected to be launched $\sim 2012$. The payload includes an $\mathrm{X}$-ray spectrometer whose science objectives include the production of global elemental abundance maps of key elements to an accuracy of $10-20 \%$, performing spatially resolved measurements of these elemental abundances, and realising high spatial resolution mapping where solar conditions permit. Achieving these objectives will allow us to address key science issues such as the origin and evolution of Mercury, the nature of surface modifications on Mercury (e.g. cratering and volcanism) and the structure and variation of the magnetosphere of Mercury

The main scientific requirements for the $\mathrm{X}$ ray spectrometer include an efficient detection in the energy range of 0.5 to $7.5 \mathrm{keV}$, and energy resolution $<250 \mathrm{eV}$, angular resolution of as little as $500 \mathrm{~m}$ and a field of up to 100 $\mathrm{km}$, depending on altitude (the very eccentric orbit will allow a periherm of $<500 \mathrm{~km}$ ). The optics will be realized by a novel microchannel plate glass pore technology, in a Wolter 1 telescope configuration [3]. With a modest $1 \mathrm{~m}$ focal length and $200 \mathrm{~mm}$ diameter optic, arcminute class resolution can be obtained in a very light weight structure. The benefit of using CS detectors for this and other planetary missions comes from the severe payload resource limits (e.g. no cooling) and intense radiation fields.

\subsection{Gamma Telescopes}

To date hard-X-ray and $\gamma$-ray imaging for astrophysics has been limited to techniques of mechanical collimation and coded masks [4]. Not only do these limit imaging resolution, but also limit the photon collection area to that of the detector frontal surface. True imaging that allows to decouple the collection area from that of the detector is necessary to make solid progress in this area of astrophysics. At such point the development of compact highly efficient detectors will be crucial in order futher to reduce internal background components. Developments in depth-graded multi-layered coatings for high energy reflectivity [5] and low mass highly nested substrates are expected shortly to demand these detector developments. 


\section{GaAs and Imaging Arrays}

\subsection{Monolithic}

Following a programme of material improvements, Owens et al [6] have reported data from a series of detectors fabricated from GaAs grown by CVPD (chemical vapour phase deposition). Characterisation of the material by photoluminescence showed very low impurity concentration $\left(10^{12} \mathrm{~cm}^{-3}\right)$. The intrinsic layers were grown on $200 \mu \mathrm{m}$ thick $\mathrm{n}^{+}$ GaAs substrates to thicknesses between 40 and $400 \mu \mathrm{m}$. P-I-N structures were made by depositing a $<5 \mu \mathrm{m} \mathrm{p^{+ }}$ layer onto the epitaxial layer, and an Ohmic contact $(\mathrm{Ni} / \mathrm{Au} / \mathrm{Ge} / \mathrm{Au})$ on the $\mathrm{n}^{+}$side. The $\mathrm{p}^{+}$layer was patterned for detector mesa and guard structures, and further deposited with a Schottky $\mathrm{Au} / \mathrm{Pt} / \mathrm{Ti}$ contact. IV characteristics measured at room temperature showed a current density less than $4 \mathrm{nAcm}^{-2}$ with a bias of $-100 \mathrm{~V}$. In Figure 1 we show the energy loss spectrum for a ${ }^{241} \mathrm{Am}$ source measured with a $\sim 1 \mathrm{~mm}^{2}$ area device, $40 \mu \mathrm{m}$ thick. The operating temperature was approximately $-20^{\circ} \mathrm{C}$ and the bias $160 \mathrm{~V}$. For comparison we have also shown in the inset data for a ${ }^{55} \mathrm{Fe}$ source. The measured energy resolution was $\sim 400 \mathrm{eV}$ FWHM at $5.9 \mathrm{keV}$, increasing to only $670 \mathrm{eV}$ at $59 \mathrm{keV}$.

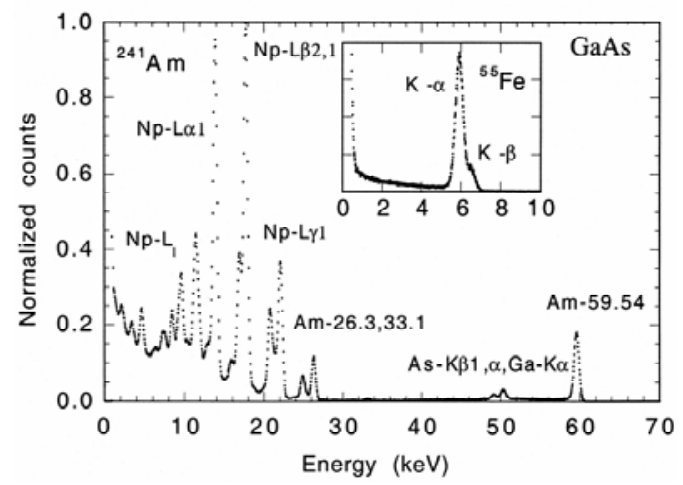

Figure $1{ }^{241}$ Am energy loss spectrum for a monolithic GaAs diode, $1 \mathrm{~mm}$ diameter and $40 \mu \mathrm{m}$ thick.

\subsection{Arrays}

Using the same material, small pixel detector arrays $\left(250 \times 250 \mu^{2}\right)$ have also been fabricated. In this case energy resolutions of only $\sim 220 \mathrm{eV}$ FWHM were achieved at an energy of $5.9 \mathrm{keV}$. This development is being continued to provide arrays with $32 \times 32$ pixels, with the eventual aim of mating these to a spectroscopic readout ASIC.
Ad interim, we have developed the technologies for thinning GaAs substrates, in order to trade off detection thicknesses and spatial resolution. Thinned substrates were then bonded to the patterned $\mathrm{p}^{+}$layer via. low temperature solder bumps to a photon-counting MEDIPIX readout ASIC [7]. This demonstrates the technology for $\mathrm{cm}^{2}$ - sized arrays to be bump-bonded with very low pixel drop outs, and demonstrate excellent imaging capabilities, albeit without the eventual required X-ray energy-resolving capability. (Figure 2)

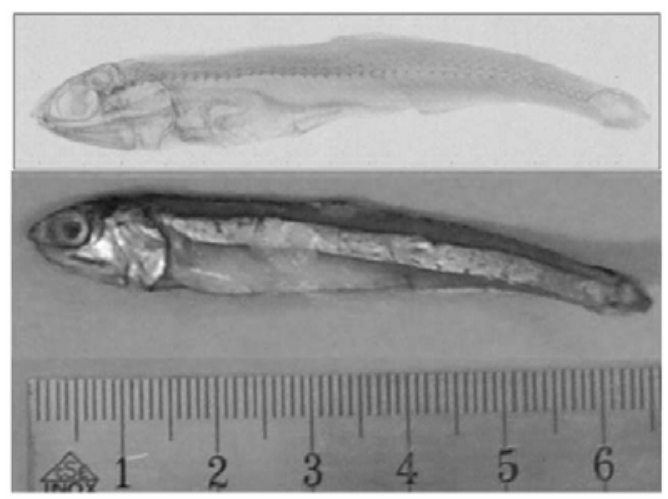

Figure 2. A 64x64 pixel (170x170 $\mu \mathrm{m}^{2}$ each) GaAs array mated to a Medipix readout array has been exposed to a standard fish sample and exposed with ${ }^{109} \mathrm{Cd}$ to produce this shadowgram, that is tiled in the horizontal direction

\section{CdZnTe}

\subsection{Planar Detector}

To date, of all candidate CS materials, the ternary compound $\mathrm{CdZnTe}$ has received most attention for hard X-ray detection applications. The addition of a few $\% \mathrm{Zn}$ to the CdTe melt increases the band-gap and bulk resistivity while reducing the dislocation densities and leakage current. However CdZnTe still suffers from relatively high densities of trapping sites compared with $\mathrm{Si}$ and $\mathrm{Ge}$ detectors, and furthermore the transport properties of electrons and holes are significantly different. To obtain acceptable energy resolution requires techniques to rely on single carrier species discrimination. For example, applying a bias to force efficient electron collection can leave events with long path length for collecting holes (typically for high energies) which then exhibit poor collection efficiencies. 


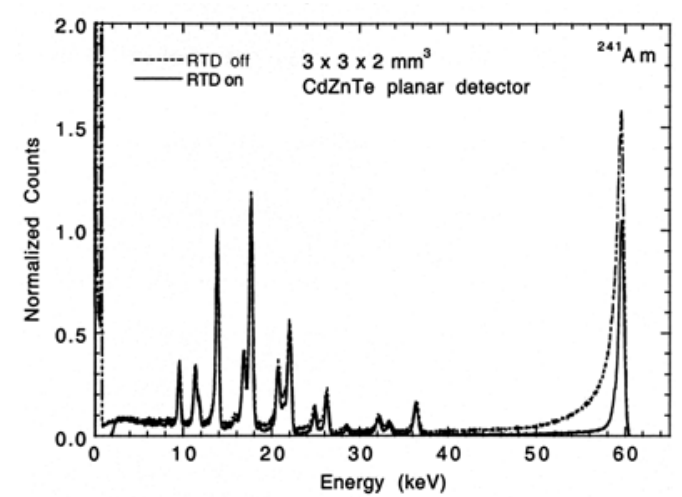

Figure 3 Comparison of ${ }^{241} \mathrm{Am}$ energy loss spectrum with and without RTD enabled in $3 \times 3 \times 2 \mathrm{~mm}$ CdZnTe detector. Hole tailing is substantially reduced but energy dependent photopeak efficiency is also reduced.

Owens et al [8] describe measurements on a $3 \times 3 \times 2 \mathrm{~mm}^{3}$ detector provided by Amptek Inc.. The detector was Peltier cooled to $\sim-30{ }^{\circ} \mathrm{C}$. Details were presented of the use of Rise Time Discrimination (RTD) to select for events corresponding with full charge collection, and how this can improve the energy resolution, but at the expense of detection efficiency (Figure 3).

The array has been mapped with a synchrotron beam at HASYLab X1 station, and in the range between $10 \mathrm{keV}$ and $100 \mathrm{keV}$, using a $\mathrm{Si}$ monochromator with $1 \mathrm{eV}$ resolution. Measurements were taken at the ESRF ID15 beam station in the range $30 \mathrm{keV}$ to $1 \mathrm{MeV}$.

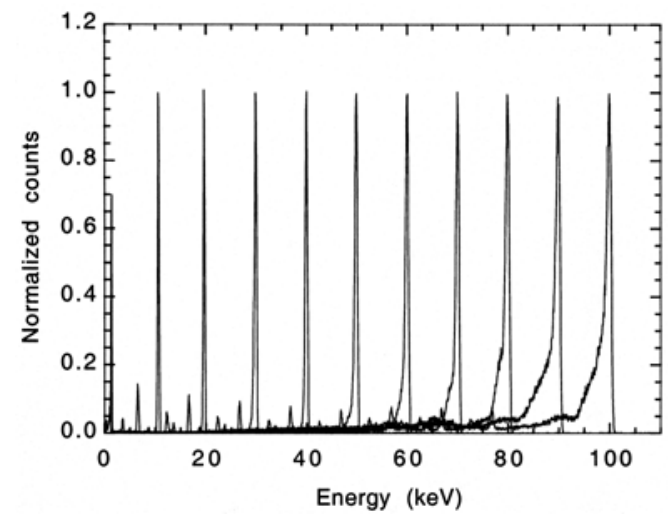

Figure 4 Composite energy spectra of monochromatic radiation. Illumination by a 20 micron spot at the centre of a $3 \times 3 \mathrm{~mm}$ array.

Figure 4 summarises the variation in hole tailing with energy. Below $50 \mathrm{keV}$ the response is almost completely a Gaussian photopeak, with continuum less than $1 \%$ of peak amplitude. Energy resolution increases from $270 \mathrm{eV} \mathrm{FWHM}$ at $5.9 \mathrm{keV}$ to $930 \mathrm{eV}$ FWHM at $59 \mathrm{keV}$ (or $700 \mathrm{eV}$ with RTD).
However the effect of RTD is to reduce the photo peak efficiency from $90 \%$ of non-RTD mode photopeak efficiency $(20 \mathrm{keV})$ to only $20 \%$ by $100 \mathrm{keV}$.

\subsection{Coplanar Grid}

An alternative approach to maintaining spectral response is the co-planar grid technique $[9,10]$ that emulates the Frisch grid employed in gaseous detectors, and where electrons drift towards the anode through the grid, and positive ions in the opposite direction. It is formed in the semiconductor detector by arranging two anode electrodes as inter-digitated grids connected to separate preamplifiers (Figure 5). The field in the detector bulk is established with a cathode bias that is a full-area contact on the opposite side. A bias is applied between the anode grids to preferentially collect charge on one set, and a subtraction circuit senses differences in signal between the anodes. Charge motion in the bulk is sensed equally and no difference signal is manifested. Charges approaching the grid create a different signal in each grid due to the bias potentials. In a uniform detector with minimal electron trapping, the difference signal should remain negligible.

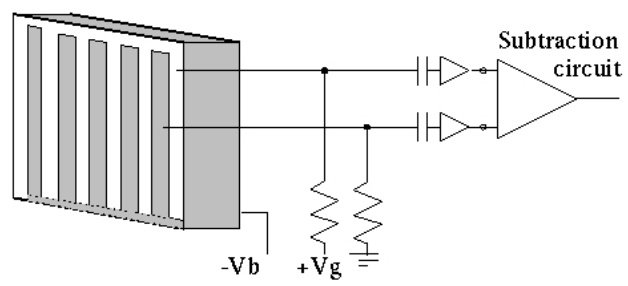

Figure 5 The coplanar grid concept - bias is provided between electrode and rear face $\left(\mathrm{V}_{\mathrm{b}}\right)$ and a small bias $\left(\mathrm{V}_{\mathrm{g}}\right)$ placed between the two inter-digitated electrodes.

A $15 \times 15 \mathrm{~mm}^{2}$ grid was formed on a 17x17x17 $\mathrm{mm}^{3}$ CdZnTe detector [11]. A cathode bias of $1.7 \mathrm{keV}$ and inter-grid bias of $60 \mathrm{~V}$ was employed. This detector was tested at the same synchrotron facilities as the planar detector. The uniformity of response is shown in Figure 6, where a spot of $180 \mathrm{keV}$ illumination has been rastered across the array. There is a very small imprint of the grid structure visible on the surface plot for peak centroid (ii).

The energy linearity and resolution were measured at a number of energies. Unlike the planar detector, the linearity is excellent up to 
more than $1 \mathrm{MeV}$. Resolution was limited by a measured system noise of $7 \mathrm{keV}$ FWHM, ranging from $8.4 \mathrm{keV} \mathrm{FWHM}$ at $20 \mathrm{keV}$ to 12.9 $\mathrm{keV}$ FWHM at $800 \mathrm{keV}$ (Figure 7).
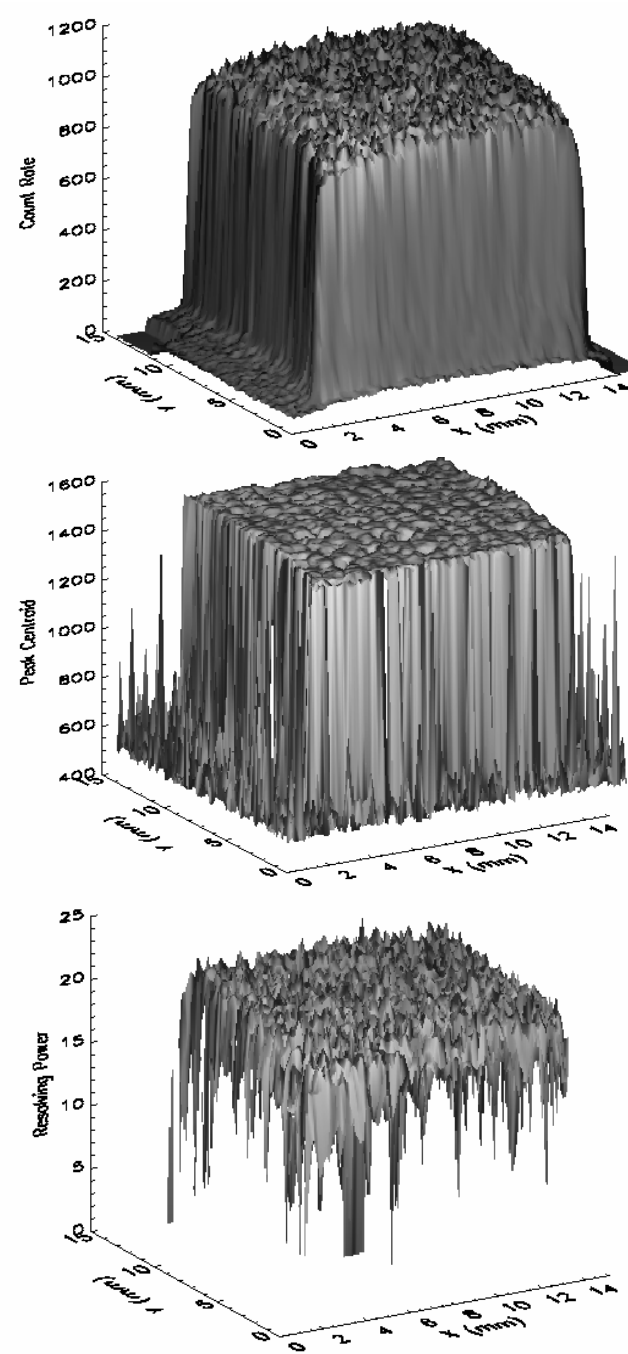

Figure $6 \mathrm{CdZnTe}$ co-planar grid detector - uniformity of response mapped with a synchrotron beam with 20 micron spot. From top to bottom (i) Count rate, (ii) gain and (iii) resolving power

These data show that CdZnTe can effectively be used as a $\gamma$-ray detector with resolutions comparable with the best scintillators. The thickness of conventional CS detectors is limited to a few $\mathrm{mm}$, here the crystal detection depth is already $>10 \mathrm{~mm}$, and the useful detection volume $>10 \mathrm{~cm}^{3}$.
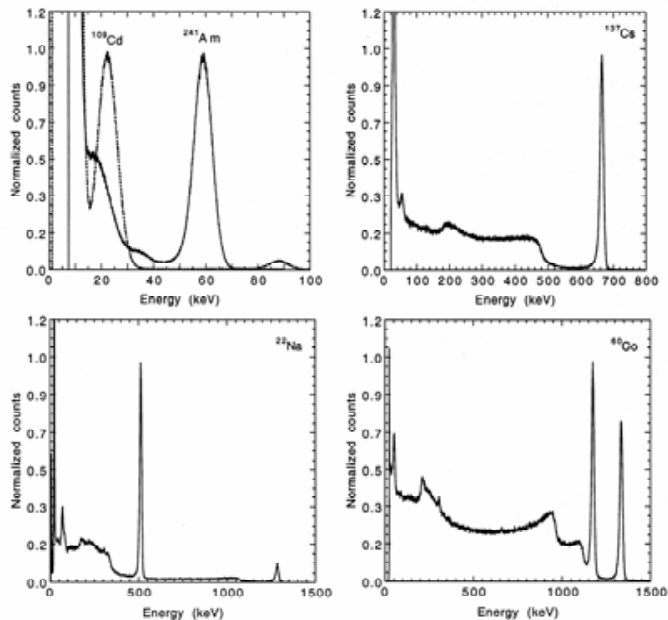

Figure 7. Measured response at room temperature of coplanar grid $\mathrm{CdZnTe}$ detector. Full area illumination of ${ }^{109} \mathrm{Cd},{ }^{241} \mathrm{Am},{ }^{137} \mathrm{Cs},{ }^{22} \mathrm{Na}$ and ${ }^{60} \mathrm{Co}$. Energy resolution varies from $8 \mathrm{keV}$ to $17 \mathrm{keV}$ FWHM for $59.5 \mathrm{keV}$ to 1.33 $\mathrm{MeV}$ energies respectively

\section{Other Gamma-ray detectors}

Indium phosphide is a CS with electronic and structural properties that are very similar to $\mathrm{Si}$, and has been the subject of extensive development for sensors for fibre-optics in the telecommunications industry. Further benefits for CS detector developments may arise from this conventional semiconductor investment.

The extremely high electron mobility makes it potentially of use for very high count rate applications (e.g. as a synchrotron reference detector). With large band gap it should be operable at room temperature, and with a density twice that of Si offers good efficiency up to $10 \mathrm{keV}$. We have demonstrated [6] energy resolution of $2.5 \mathrm{keV} \mathrm{FWHM}$ at $5.9 \mathrm{keV}$ increasing to $\sim 9 \mathrm{keV}$ FWHM at $59 \mathrm{keV}$ (Figure 8)

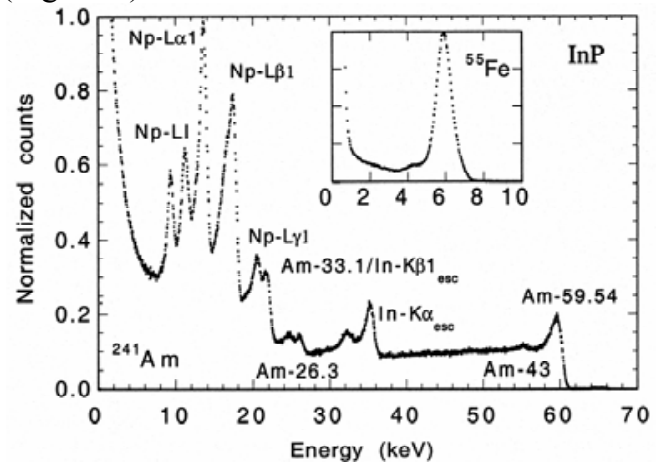

Figure 8. Energy loss spectrum from ${ }^{241} \mathrm{Am}$ observed with InP diode $180 \mu \mathrm{m}$ thick and $2 \mathrm{~mm}$ in diameter $\left({ }^{55} \mathrm{Fe}\right.$ spectrum in the inset) 
For yet harder energies, effort has been expended in developing denser compounds, including $\mathrm{Pb}, \mathrm{Bi}$ and $\mathrm{Tl}$ compound semiconductors. Transport properties of $\mathrm{PbI}$ are too poor to admit detector thicknesses much greater than 10's $\mu \mathrm{m}$. Better results have recently been obtained with $\mathrm{TlBr}$ [12], where resolutions of 1.8 and $3.3 \mathrm{keV}$ FWHM at 5.9 and $59 \mathrm{keV}$ respectively have been recorded for detectors of dimension $2.8 \times 2.8 \times 0.8 \mathrm{~mm}^{3}$ and operated at room temperature (Figure 9). Improvements occur with cooling, but there was evidence of gain and resolution changes that occurred with count rate (so-called polarisation effects)

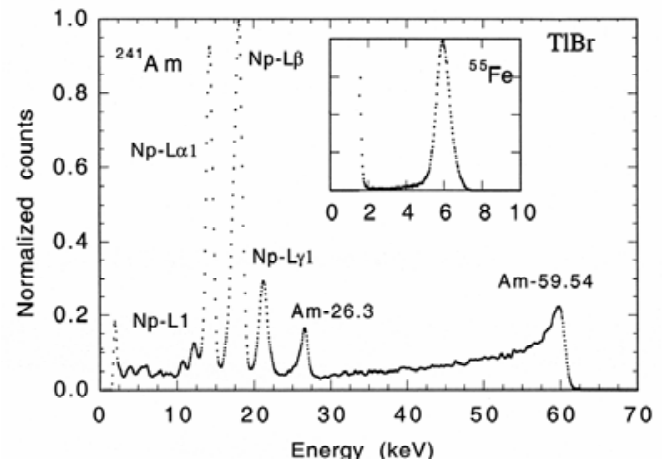

Figure 9. Energy loss spectrum from ${ }^{241}$ Am observed with $\mathrm{TlBr}$ diode $800 \mu \mathrm{m}$ thick and $2.8 \times 2.8 \mathrm{~mm}$ in size $\left({ }^{55} \mathrm{Fe}\right.$ spectrum in the inset)

\section{Narrow band Gap}

We comment finally on an orthogonal approach that we are now investigating, namely to develop low band-gap materials. Despite the disadvantage of requiring substantially lower temperatures, the corresponding increase in number of electronhole pairs liberated per absorbed photon allows an improvement in photoelectron statistics and enhanced energy resolution. Recognising that $\mathrm{InSb}$ and $\mathrm{HgCdTe}$ have been successfully used for IR focal planes [13], we believe it is possible to develop the material for X-ray detection by following a similar programme of material processing improvements. The improvement in spectroscopic readout ASIC will also be necessary to ensure low enough noise to obtain Fano-limited resolution at sub$\mathrm{keV}$ energies as well. To date there is only a reported detection of $\mathrm{MeV}$ alpha particles with InSb [14], and IR focal plane detectors have low resistivity and very thin material that is mounted on semi-opaque substrates.

\section{References}

1. D.H. Lumb, G.D. Berthiaume, D.N. Burrows et al Exptl Astron 2 (1999) 179

2. R. Schulz, Proceedings 35th COSPAR Scientific Assembly. July 2004, Paris, France., p.1289

3. M. Beijersbergen, M. Bavdaz, A. Peacock et al Proc. SPIE Vol. 4012, (2000) p. 218-224

4. G.K. Skinner Proceedings $22^{\text {nd }}$ Moriond Astrophysics Workshop, “The Gamma-Rauy Universe”, Ed A Goldwurm, pub The Gioi, Vietnam

5. J. Koglin, F. Christensen, J. Chonko, et al Proc SPIE 4851 (2003) 607241

6. A. Owens, A. Peacock, M. Bavdaz Proc SPIE 4851 (2003) 1059

7. A. Owens, H. Andersson, M Campbell, et al Proc SPIE 5501 (2004)

8. A Owens, T. Buslaps, C Erd, et al, submitted to Nucl Inst Meth Phys Res A

9. P.N. Luke IEEE Trans Nucl Sci. NS 32 (1985) 556

10. P.N. Luke IEEE Trans Nucl Sci NS 42 (1995) 207

11. A. Owens, T Buslaps, V Gostilo et al submitted to Nucl inst Meth Phys Res A

12 V. Gostilo, , M. Bavdaz et al Nucl Inst Meth Phys Res A 509 (2003) 47

13. A.W. Hoffman, E. Corrales, P. Love Proc SPIE 5499 (2004) 59

14. I. Kanno, F. Yoshihara, R. Nouchi, et al Rev Sc Inst 74 (2003) 3968 